\title{
Tomographie par émission de positrons dans la prise en charge des carcinomes épidermoïdes des VADS
}

\author{
Siham Kerrary ${ }^{\star}$, Jacques Charles Bertrand, Didier Ernenwein \\ Service de Chirurgie maxillo-faciale et Stomatologie, Hôpital de la Pitié-Salpêtrière, Université Pierre et Marie-Curie, \\ Paris 6, France
}

(Reçu le 7 avril 2010, accepté le 26 avril 2010)

\section{Mots clés :}

TEP-TDM /

carcinomes des voies aérodigestives supérieures
Key words:

PET-CT /

head and neck carcinomas

\begin{abstract}
Résumé - Le carcinome épidermoïde représente la forme la plus fréquente des cancers des voies aérodigestives supérieures (VADS). L'anatomie complexe de la région cervicofaciale rend son exploration assez difficile. Si l'imagerie conventionnelle permet d'obtenir des informations topographiques intéressantes, la médecine nucléaire avec la tomographie par émission de positrons (TEP) au [ $\left.{ }^{18} \mathrm{~F}\right]$-fluoro-2-désoxy-Dglucose $\left({ }^{18} \mathrm{~F}-\mathrm{FDG}\right)$ constitue un progrès pour le bilan d'extension dans les aires ganglionnaires et la détection de néoplasies synchrones. Sa place est appelée à devenir primordiale pour la recherche de lésions résiduelles et la détection précoce des récidives. L'évaluation de la réponse à la chimiothérapie et l'optimisation de la radiothérapie (champs et dosimétrie) constituent également des applications prometteuses car la technique de la TEP est en évolution constante.
\end{abstract}

\begin{abstract}
Positron emission tomography in head and neck squamous cell carcinomas. Squamous cell carcinoma is the most frequent of head and neck cancers. The complex anatomy of head and neck areas makes more difficult its exploration. If the conventional imaging provides interesting topographic informations, the contribution of the nuclear medicine through positron emission tomography (PET) with $\left[{ }^{18} \mathrm{~F}\right]$-fluoro-2-deoxy-D-glucose is efficient for staging lymph nodes and detection of synchronous neoplasias. Besides, its place is also known to be essential for detecting residual lesions and early detection of recurrences. Assessment of tumor response to treatment with chemotherapy and optimization fields and dosimetry in radiotherapy are also proving to be full of promising applications especially because the technique by PET is in constant evolution.
\end{abstract}

\section{Généralités et épidémiologie}

Les carcinomes épidermoïdes de la cavité buccale représentent environ $95 \%$ des cancers des voies aérodigestives supérieures (VADS). Les facteurs de risque sont dominés par l'intoxication éthylotabagique, également responsable de la survenue d'une deuxième localisation [1].

À l'instar de ceux de l'amygdale, certains carcinomes épidermoïdes de la cavité buccale (cancers linguaux essentiellement) peuvent être associés aux Human Papilloma Virus (HPV) notamment à l'HPV16; l'incidence varie en fonction des séries rapportées. Ces carcinomes épidermoïdes surviennent en règle générale chez des patients plus jeunes, ne présentant pas d'intoxication éthylotabagique. Dans les carcinomes épidermoïdes de la cavité buccale, le pourcentage de patients

\footnotetext{
^Correspondance : sihamkerrary@hotmail.fr
}

chez lesquels on retrouve du HPV16 intratumoral par PCR varie de 5 à $15 \%$ [2].

Le bilan préthérapeutique obéit à une méthodologie précise comportant l'analyse des symptômes, un examen clinique local et locorégional (aires ganglionnaires cervicofaciales) : il doit être soigneux, méthodique, complet, sans oublier la palpation systématique de la langue. L'examen clinique doit être complété par une biopsie de la tumeur car l'examen anatomopathologique constitue le seul moyen diagnostique fiable.

Avant tout traitement, un examen radiologique doit compléter le bilan; il permet d'explorer les aires ganglionnaires et de rechercher d'éventuelles métastases à distance. La classification TNM, effectuée à partir des éléments obtenus, permet d'établir un plan de traitement adapté à chaque cas. Le bilan d'extension locorégional comporte un examen tomodensitométrique (TDM) à haute résolution pour visualiser les tumeurs de petite taille, leur extension osseuse éventuelle, ainsi que 
les adénopathies infracliniques. Ce bilan peut être complété par l'imagerie par résonance magnétique (IRM) conventionnelle qui apporte des informations sur la néovascularisation et l'infiltration tumorales. Le bilan d'extension à distance se limite le plus souvent à une TDM thoraco-abdomino-pelvienne.

La panendoscopie sous anesthésie générale est également systématique. L'ensemble des VADS est examiné à la recherche d'une seconde localisation. Une biopsie de la lésion est réalisée si elle n'a pas été faite auparavant. La palpation de la cavité buccale, de l'oropharynx et des aires ganglionnaires est répétée pendant l'anesthésie générale.

Cependant, quelle que soit la résolution atteinte, les techniques d'imagerie sont purement morphologiques et elles ne permettent guère de préciser la nature des lésions. En effet, les lésions malignes ne présentent pas de densité particulière à la TDM ou de signal spécifique en IRM, même après l'injection par voie intraveineuse d'un produit de contraste. Seule l'augmentation de la taille de la lésion ou des ganglions peut faire suspecter un processus malin. Il existe donc une place pour une technique d'imagerie utilisant les caractères biochimiques ou moléculaires des cellules malignes. La tomographie par émission de positions (TEP) au ${ }^{18} \mathrm{~F}$-fluorodésoxyglucose $\left({ }^{18} \mathrm{~F}-\mathrm{FDG}\right)$ est basée sur cette approche.

\section{Technique de la TEP/TDM}

\section{Principe}

Il s'agit d'un examen d'imagerie de type scintigraphie. En injectant un analogue de glucose, utilisé comme radiomarqueur, on obtient avec la TEP une image caractérisant l'activité métabolique de la lésion. Le radiomarqueur se fixe sur les zones où le métabolisme glucidique anaérobie est important, comme dans les cellules cancéreuses [3]. Par détection externe, on observe l'image de sa distribution in vivo après un délai dépendant de la physiologie du traceur. En effet, ce traceur est marqué par un atome radioactif (fluor, carbone, azote...) qui émet des positrons dont l'annihilation produit elle-même deux photons. La détection de la trajectoire de ces photons par la caméra TEP permet de mettre en œuvre de multiples corrections physiques destinées à faire de l'image réalisée une reproduction la plus exacte possible de la distribution réelle du traceur chez le patient, et ce avec une bonne résolution spatiale. L'analyse des images de la TEP est surtout visuelle et qualitative, mais il est possible de calculer divers indices chiffrés exprimant la quantité de traceur fixée ou accumulée dans une structure anatomique ou une lésion. L'indice le plus fréquemment utilisé est la standardized uptake value (SUV), obtenu en rapportant l'activité du traceur fixé sur la tumeur à l'activité totale du traceur injecté, en tenant compte du poids du patient. La caméra TEP « petscan » permet, après acquisition des données par un balayage longitudinal, de construire des images dans les trois plans de l'espace et sous la forme de projections volumiques. La dernière génération de tomographes est couplée à un tomodensitomètre au sein du même appareil, ce qui permet de superposer l'image métabolique sur l'image anatomique.

Le traceur émetteur de positrons le plus utilisé en imagerie clinique est le ${ }^{18} \mathrm{~F}-\mathrm{FDG}$. Pour sa pénétration dans les cellules, il utilise le même transporteur membranaire que le glucose. Le critère diagnostique de malignité est donc l'existence d'une hyperfixation témoignant de l'augmentation de l'activité métabolique [4].

\section{Réalisation de l'examen}

Pour l'examen, le patient doit être à jeun depuis 6 heures et bien hydraté pour permettre l'élimination rénale du traceur. La réalisation de l'examen est longue, de l'ordre de 2 à 3 heures. Hormis l'injection intraveineuse du traceur, il s'agit $d^{\prime} u n$ examen non invasif. Aucun effet indésirable du ${ }^{18} \mathrm{~F}-\mathrm{FDG}$, ni aucun phénomène allergique n'ont été rapportés après plus de vingt ans d'utilisation. Le patient doit être au repos, au lit ou dans un fauteuil, afin d'éviter toute activité musculaire susceptible d'entraîner une hyperfixation non spécifique pendant l'heure qui précède l'injection du traceur, et pendant la période de fixation qui dure 1 à 2 heures. La prescription $d^{\prime}$ une prémédication myorelaxante et anxiolytique est parfois conseillée.

Après cette période de repos et d'attente, le patient est installé sur la table d'examen en décubitus dorsal, les bras placés au-dessus de la tête ou le long du corps. L'acquisition des images corps entier (champ dédié de la région cervicofaciale complété par un balayage corps entier) se fait en trente minutes environ avec un appareil hybride TEP-TDM de dernière génération. Cette durée peut encore être réduite à une vingtaine de minutes avec les derniers appareils utilisant par exemple la technologie de temps de vol.

L'irradiation pour l'examen TEP-TDM est du même ordre que celle délivrée lors d'une TDM à visée diagnostique [5]. L'épaisseur des coupes est, en général, de l'ordre de 3 à $4 \mathrm{~mm}$. L'interprétation est réalisée sur une console de traitement avec lecture des coupes coronales, sagittales et transaxiales reconstruites. Le résultat de l'examen est rendu sous forme d'images en couleur, résultant de la fusion des données scannographiques et scintigraphiques, donnant une vision fonctionnelle et dynamique du métabolisme de l'organe ou de la lésion observés. Le seuil de détection varie de 6 à $8 \mathrm{~mm}$ selon la quantité de ${ }^{18} \mathrm{~F}-\mathrm{FDG}$ fixé.

\section{Indications de la TEP-FDG et de la TEP-TDM}

Elle peut être utilisée pour le diagnostic, le bilan initial $[5,6]$, la surveillance après le traitement [7] et la mise en évidence des récidives [8].

\section{Lors du bilan initial}

Il existe deux possibilités : soit la tumeur primitive est identifiée, soit il s'agit d'une adénopathie sans porte d'entrée. 
Si la tumeur est accessible à l'examen direct et à la biopsie, le diagnostic est connu : la TEP-FDG n'a pas d'indication. Cependant, face à une lésion cliniquement ou radiologiquement suspecte, non confirmée par une première biopsie, elle peut aider à déterminer le siège de la nouvelle biopsie; elle permet donc d'augmenter les chances de succès. La TEP-FDG ne fournit pas d'indication sur la nature histologique de la lésion, mais l'intensité de la fixation est corrélée à l'activité de la prolifération cellulaire. La valeur du SUVmax de la lésion primitive lors du bilan initial constitue un facteur pronostique : une valeur élevée du SUVmax (SUVmax supérieur à 6 ou à 8) incite à surtraiter les lésions les plus hypermétaboliques. Selon le SOR (Standards, Options et Recommandations) concernant l'utilisation de la TEP-FDG en cancérologie paru en 2003, l'étude quantitative de la fixation du ${ }^{18} \mathrm{~F}-\mathrm{FDG}$ par une tumeur maligne des VADS a une valeur pronostique, aussi bien pour la survie que pour le risque de récidive après traitement [9].

Les métastases ganglionnaires présentent également un hypermétabolisme, sans relation avec la taille du ganglion. Cela constitue une différence importante avec les techniques d'imagerie qui ne fournissent que des renseignements sur la taille et la forme du ganglion. Or, à un stade précoce, le ganglion métastatique ne présente pas immédiatement une augmentation de taille. De plus, un ganglion peut être augmenté de taille lors d'un processus inflammatoire ou infectieux. Cela explique la différence de performances retrouvées dans la littérature entre la TEP et l'imagerie radiologique. Ainsi, pour l'évaluation de l'extension ganglionnaire, de nombreuses études ont montré les avantages de la TEP-FDG par rapport à l'imagerie classique lors du bilan d'extension initial; cette supériorité est encore plus évidente avec les appareillages hybrides TEP-TDM [10].

En plus du bilan d'extension ganglionnaire locorégional, l'acquisition corps entier permet d'explorer les autres aires ganglionnaires, les tissus ou les organes fréquemment concernés par les métastases comme les poumons, le squelette et le foie, avec une très grande aptitude à la différentiation entre lésions bénignes et malignes (deuxième localisation et métastases), sous réserve que les lésions aient un volume suffisant. Ainsi des métastases à distance, méconnues par les techniques d'imagerie, sont découvertes avec la TEP-FDG dans 10 à $20 \%$ des cas [10]. La technique présente un intérêt pour la recherche, dans le même temps, d'une deuxième lésion maligne synchrone éventuellement due aux mêmes facteurs de risque (poumons, œsophage...); elle est mise en évidence dans $15 \%$ à $20 \%$ des cas [10].

Pour les adénopathies métastatiques sans porte d'entrée, qui représentent 2 à $9 \%$ des carcinomes épidermoïdes de la région cervicofaciale, la TEP-FDG pratiquée après les explorations conventionnelles (TDM, IRM, endoscopie) négatives permet d'identifier la tumeur primitive dans $30 \%$ des cas en moyenne (8 à $54 \%$ selon les études). Elle constitue donc une indication majeure de la TEP-FDG pour découvrir la tumeur primitive et déterminer le siège de la biopsie. Si la TEP est négative, une panendoscopie avec des biopsies étagées est indiquée. La TEP-TDM a une sensibilité ( $86 \%$ à $100 \%$ ) et une spécificité (69\% à $87 \%)$ supérieures à celles de la TDM (respectivement $67 \%$ à $82 \%$ et $25 \%$ à $56 \%$ ) pour la détection des tumeurs primitives et des métastases ganglionnaires [11].

\section{Détection précoce des récidives}

La TEP-TDM a une sensibilité variant de $93 \%$ et $100 \%$ pour la détection des récidives de la tumeur primitive, des métastases ganglionnaires locorégionales et des métastases à distance. Elle est réalisée trois mois après la fin du traitement, délai nécessaire pour éviter un trop grand nombre de faux positifs induits par la cicatrisation des lésions radiques, et de faux négatifs liés au caractère microscopique de certaines lésions résiduelles. Ce délai est suffisant pour permettre la reprise du développement du cancer persistant après le traitement [10].

La TEP-TDM est réalisée après un examen clinique et une TDM. Elle permet d'identifier les patients ne présentant pas de récidive et, dans ce cas, on se limite à une surveillance clinique du patient, sans avoir à refaire une panendoscopie. Elle permet aussi d'éviter une nouvelle biopsie, souvent peu contributive, qui peut de plus favoriser le développement d'une radionécrose $[10,12]$.

L'endoscopie et la biopsie sont donc uniquement réalisées lorsque la TEP-TDM est positive [12].

\section{Étude de la réponse au traitement}

La TEP-FDG est particulièrement utile pour évaluer l'efficacité du traitement après radiothérapie et/ou chimiothérapie. Habituellement, on évalue l'efficacité du traitement sur la diminution du volume des lésions sur des TDM successives. La TEP-FDG peut mettre en évidence la persistance d'une fixation d'intensité constante ou accrue dans une lésion de volume plus faible. L'imagerie métabolique fournit une information précoce sur les effets de la chimiothérapie. On peut ainsi apprécier la réponse de la tumeur à la chimiothérapie dès le premier cycle; cela permet d'éviter la poursuite d'un traitement inefficace et coûteux [13].

\section{Optimisation des champs de radiothérapie}

Avec la TEP-TDM, il est devenu possible d'intégrer les informations métaboliques fournies par la TEP pour déterminer les champs de radiothérapie. Selon plusieurs publications, la prise en compte des données de la TEP-FDG a entraîné un changement de protocole thérapeutique dans environ $20 \%$ des cas. Ces changements concernent plus le traitement des aires ganglionnaires (aboutissant le plus souvent à une augmentation qu'à une diminution des champs d'irradiation initialement prévus) que celui de la tumeur [14]. Cette application est encore du domaine de la recherche; d'autres études sont nécessaires pour mieux préciser les limites des lésions fournies pas la TEP-TDM. 


\section{Limites d'utilisation et contre-indications}

Il est important de souligner le taux important de faux positifs avec la TEP-TDM, quelle que soit son indication. Les causes sont multiples et principalement dues au manque de spécificité du ${ }^{18} \mathrm{~F}$-FDG pour le tissu tumoral. Les faux positifs peuvent être dus à une contracture musculaire unilatérale, à une activation des muscles phonatoires et à des mouvements de déglutition lors de la phase de fixation du ${ }^{18} \mathrm{~F}-\mathrm{FDG}$, une accumulation de salive, un papillome de l'amygdale, une trachéotomie, un cathéter... D'une manière générale, toute réaction inflammatoire (lésion postradique, tissu en cours de cicatrisation, foyer infectieux) est susceptible de fixer le ${ }^{18} \mathrm{~F}-\mathrm{FDG}$.

Par ailleurs, on peut observer fréquemment un taux élevé de fixation physiologique de ce radiotraceur dans certaines régions anatomiques. Une étude réalisée par Nakamoto et al. [15] sur 78 patients en bonne santé a montré une fixation physiologique importante sur les amygdales et le voile du palais, une fixation intermédiaire sur les glandes salivaires (parotides, submandibulaires) et sur les cordes vocales. Cette hyperfixation physiologique n'est pas assimilée à un faux positif mais elle a un rôle délétère en gênant l'interprétation de l'examen car elle est à l'origine de nombreux artefacts. Cependant le nombre de résultats faux positifs a été nettement réduit avec la TEP-TDM qui permet une meilleure localisation anatomique des zones hyperfixantes. Il existe également des faux négatifs : ils sont principalement liés à la présence de tissu nécrotique autour du tissu tumoral, à la petite taille de la tumeur et à l'hypermétabolisme physiologique de la région cervicofaciale. Cet hypermétabolisme et le degré de discrimination de la caméra TEP rendent aléatoire la détection des tumeurs de moins de $6 \mathrm{~mm}$ par la TEP-TDM [10].

Enfin, la TEP, même couplée à la TDM, est moins performante qu'une TDM millimétrique conventionnelle mais elle ne nécessite pas l'injection de produit de contraste iodé. La TDM et éventuellement l'IRM restent donc indispensables, notamment pour évaluer l'infiltration en profondeur et la vascularisation de la tumeur. C'est en prenant le patient dans sa globalité que la TEP-TDM présente un intérêt majeur [12].

La TEP-TDM trouve également ses limites dans la détection des récidives, en particulier chez les patients où les lambeaux utilisés pour la reconstruction entraînent d'importantes modifications de l'anatomie. La détection précoce d'une récidive est pourtant cruciale afin de pouvoir envisager une chirurgie de rattrapage. La seule véritable limite de l'examen au ${ }^{18} \mathrm{~F}$-FDG est le diabète non équilibré car l'hyperglycémie lors de l'injection du traceur réduit considérablement sa fixation dans les lésions malignes et diminue la sensibilité de l'examen. La prescription d'insuline lors de l'injection n'est pas recommandée car elle entraîne une accumulation du traceur dans les muscles squelettiques, ce qui génère d'importants artefacts et réduit la disponibilité du traceur et donc sa fixation sur les éventuelles lésions malignes.

L'utilisation de la TEP chez la femme enceinte constitue une contre-indication relative et elle doit être discutée en fonction du contexte clinique, comme pour tout examen d'imagerie utilisant des rayonnements ionisants. En période d'allaitement, l'allaitement doit être interrompu pendant plusieurs heures.

\section{Conclusion}

La TEP-FDG est un examen fiable pour la prise en charge des carcinomes épidermoïdes des VADS. Elle constitue un complément pour le bilan d'extension ganglionnaire classique, et pour la détection précoce et tardive des récidives locales. Toutefois, elle manque de précision pour la localisation anatomique. La TEP-TDM, en réalisant la fusion des images de la TEP-FDG et de la TDM, permet d'obtenir des données plus précises sur l'activité métabolique d'une tumeur et de ses métastases, et sur leur localisation anatomique.

NDLR : Après la TEP-TDM, on a envisagé la TEP-IRM mais la puissance du champ magnétique de l'IRM, en perturbant l'électronique du scanner PET, a longtemps constitué un obstacle à la réalisation de ce nouvel appareil hybride. Les progrès technologiques ont permis de lever cet obstacle et deux appareils hybrides PET-IRM (l'un au Mount Sinai Hospital à New York, l'autre à l'Hôpital cantonal de Genève) sont en cours d'évaluation.

\section{Références}

1. Legent F, Narcy P, Beauvillain C, Bordure P. Cancers des voies aérodigestives supérieures : généralités. Masson, Paris, 2003.

2. Roncin L, Touzalin A, Fleury HJ. Papillomavirus humains et tumeurs des voies aérodigestives supérieures. Lettre Infectiol 2009;24:19-27.

3. Schechter NR, Gillenwater AM, Byers RM, Garden AS, Morrison WH, Nguyen LN, Podoloff DA, Ang KK. Can positron emission tomography improve the quality of care for head and neck cancer patients ? Int J Radiat Oncol Biol Phys 2001;51:4-9.

4. Hanasono MM, Kunda LD, Segall GM, Ku GH, Terris DJ. Uses and limitations of FDG positron emission tomography in patients with head and neck cancer. Laryngoscope 1999;109:880-5.

5. Schmid DT, Stoeckli SJ, Bandhauer F, Huguenin P, Schmid $S$, von Schulthess GK, Goerres GW. Impact of positron emission tomography on the initial staging and therapy in locoregional advanced squamous cell carcinoma of the head and neck. Laryngoscope 2003;113:888-91.

6. Ha PK, Hdeib A, Goldenberg D, Jacene H, Patel P, Koch W, Califano J, Cummings CW, Flint PW, Wahl R, Tufano RP. The role of positron emission tomography and computed tomography fusion in the management of early stage and advanced stage primary head and neck squamous cell carcinoma. Arch Otolaryngol Head Neck Surg 2006;132:12-6.

7. Goerres GW, Schmid DT, Bandhauer F, Huguenin PU, von Schulthess GK, Schmid S, Stoeckli SJ. Positron emission tomography in the early follow-up of advanced head and neck cancer. Arch Otolaryngol Head Neck Surg 2004;130:105-9.

8. Kunkel M, Förster GJ, Reichert TE, Jeong JH, Benz P, Bartenstein $\mathrm{P}$, Wagner W, Whiteside TL. Detection of recurrent oral squamous 
cell carcinoma by [18F]- 2-fluorodeoxyglucose-positron emission tomography: implications for prognosis and patient management. Cancer 2003;98:2257-65.

9. Bourguet $P$, groupe de travail SOR TEP-FDG. Standards, options and recommandations for the use of PET-FDG in cancerology. Results in cancer of the upper aerodigestive tract. Bull Cancer 2003;90:567-79.

10. Ryan WR, Fee WE Jr, Le QT, Pinto HA. Positron-emission tomography for surveillance of head and neck cancer. Laryngoscope 2005; 115:645-50.

11. Gutzeit A, Antoch G, Kühl H, Egelhof T, Fischer M, Hauth E, Goehde S, Bockisch A, Debatin J, Freudenberg L. Unknown primary tumors: detection with dual-modality PET/CT-initial experience. Radiology 2005;234:227-34.
12. Lonneux M, Lawson G, Ide C, Bausart R, Remacle M, Pauwels S. Positron emission tomography with fluorodeoxyglucose for suspected head and neck tumor recurrence in the symptomatic patient. Laryngoscope 2000;110:1493-7.

13. Kostakoglu L, Goldsmith SJ. PET in the assessment of therapy response in patients with carcinoma of the head and neck and of the esophagus. J Nucl Med 2004;45:56-68.

14. Heron DE, Andrade RS, Flickinger J, Johnson J, Agarwala SS, Wu A, Kalnicki S, Avril N. Hybrid PET-CT simulation for radiation treatment planning in head and neck cancers: a brief technical report. Int J Radiat Oncol Biol Phys 2004;60:1419-24.

15. Nakamoto Y, Tatsumi M, Hammoud D, Cohade C, Osman MM, Wahl RL. Normal FDG distribution patterns in the head and neck: PET/CT evaluation. Radiology 2005;234:879-85. 